\title{
4G NETWORK PERFORMANCE ANALYSIS FOR REAL-TIME TELEMETRY DATA TRANSMITTING TO MOBILE AGRICULTURAL ROBOT
}

\author{
Andris Roberts, Aldis Pecka \\ Latvia University of Life Sciences and Technologies, Latvia \\ andris.roberts@tvnet.lv, aldis.pecka@1lu.lv
}

\begin{abstract}
Since the beginning of civilization transportation has been one of the most important problems. Even though today's vehicles carry cargoes more than a thousand kilometres far, small goods at short distances are still transported on small wheels or on small motor vehicles. With the help of such robots one person is able to drive multiple robots, which reduces labour costs, and therefore requires remotely controlled robots. Mobile equipment management is often built up by individual infrastructures, while mobile operators are now able to provide stable and fast internet connections which performance limits are unused. Nowadays, the availability of mobile internet is provided to $99 \%$ from the territory of the country. Existing resources and the full use of infrastructure are one of the cornerstones of our time, so it would be useful to make the most of the opportunities offered by the mobile communications. The main goal of the article is to analyse whether the $4 \mathrm{G}$ mobile data network is capable of providing remote management of mobile agricultural robots. In the article the existing mobile robot management systems are analysed. Flow rate checks have been performed, but no data flow stability tests have been performed to ensure the management of mobile agriculture robots. There has been performed a $4 \mathrm{G}$ mobile network data flow stability analysis. The article explores the real-time requirements for providing remote control of agricultural robots. The article outlines the results of the analysis.
\end{abstract}

Keywords: 4G mobile network, agriculture robots, real-time.

\section{Introduction}

Nowadays, one of widely used broadband mobile wireless network technologies is Fourth Generation (4G) network [1]. 4G Technology in Latvia can provide up to $55 \mathrm{Mbps}$ download speed [2]. For real-time systems there is a need for $10-20 \mathrm{Mbps}$, but there is a condition that the Internet connection needs to be stable.

To make a mobile agricultural control system a small but powerful data-processing tool or microcomputer is needed. Raspberry Pi 3 is a useful tool to make the control system of a mobile agricultural robot [3].

For qualitative mobile agricultural robot control there is a need for video stream. If video stream is with latency, then there can be a problem for accurate control. There are many different methods for measuring the delay of streaming video. The easiest way is to observe both video capturing and video displaying and compare them manually by inspecting triggers in the video. However, there are automated tools such a vDelay [4]. To count the system as a real-time system latency to transfer data should be less than $400 \mathrm{~ms}$ [5].

When transmitting data over the Internet, one has to think about data security so that someone cannot intercept data and use them abusively. One of the easiest ways to protect your data transfer is to use VPN. [6]. When you connect your computer (or another device, such as a smartphone or tablet) to a VPN, the computer acts as if it is on the same local network as the VPN. All your network traffic is sent over a secure connection to the VPN. Because your computer behaves as if it is on the network, this allows you to securely access local network resources even when you are on the other side of the world.

Because of the advantages of Bluetooth, such as low costs and low power and nature can be pointed to different directions, parts of Bluetooth have been integrated into various types of mobile devices such as mobile phones [7] and systems on WLAN.

WLAN have changed the interaction manner through wire line between operators and robots in the past. The work area of robots has not been influenced by the availability of lineate pavement anymore. But these systems work on short distances. Table 1 shows comparison of wireless technologies in detail. 
Comparison of wireless technologies [8]

\begin{tabular}{|l|c|c|c|}
\hline Types of Wireless Technology & Range & Frequency Band(s) & Data Rate, Mbps \\
\hline Bluetooth & $100 \mathrm{~m}$ & $2.4 \mathrm{Ghz}$ & 1.5 \\
\hline Wi-fi & & & \\
IEEE 802.11b & $100-300 \mathrm{~m}$ & $2.4 \mathrm{Ghz}$ & $11-100$ \\
IEEE 802.11g & & & \\
IEEE 802.11n & & & 21.6 \\
\hline 3G & Global & $2100 \mathrm{Mhz}$ & $21-150$ \\
\hline 4G & Global & $800-1800-2600 \mathrm{Mhz}$ & \\
\hline
\end{tabular}

\section{Materials and methods}

Tests are carried out using DELL Vostro 15 portable computer with the following hardware configuration: Intel® I5-7200U CPU @2.5 GHz, 8.00 GB DDR4 RAM, 224 GB Solid State drive. Computer is running with Microsoft Windows 10. All necessary up-to date drives where installed on the operating system.

4G mobile internet modem Huawei E5377s-32 with Tele2 mobile provider SIM. This modem support LTE FDD / LTE TDD / UMTS / HSUPA / HSPA +GSM / GPRS / EDGE communication system and can provide internet speed - LTE FDD $150 \mathrm{Mbps} /$ LTE TDD $112 \mathrm{Mbps}$.

Raspberry Pi 3 model B - Quad Core 1.2GHz Broadcom BCM2837 64 bit CPU, 1GB RAM, BCM43438 wireless LAN and Bluetooth Low Energy (BLE) on board ,40-pin extended GPIO, 4 USB 2 ports, 4 Pole stereo output and composite video port, CSI camera port for connecting a Raspberry Pi camera, Micro SD port for loading your operating system and storing data. [9] Raspberry Pi NoIR Infrared Camera Board v1.3 (5 MP, 1080p). 32 GB Samsung SDHC EVO Memory Card-Up to 48 MB per second transfer speed.

For security and for connection OpenVPN with self-signed certificate was used. [10] This software made a Virtual Private Network (VPN) and has included security certificates. The VPN ensures that all devices are on the same subnet, so the control computer can easily communicate with Raspberry and get data from there. The whole scheme of logical connection can be seen in Figure 1.

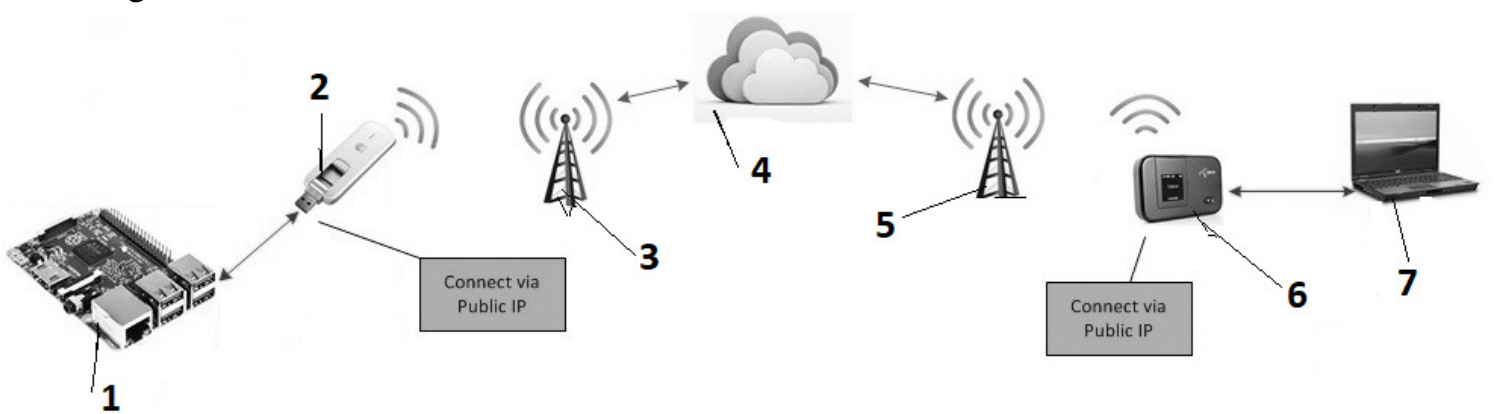

Fig. 1. Logical connection 1 - Raspberry Pi 3;2-4G modem; 3 - mobile operator; 4 - server; 5 - Internet provider; 6 - local area network; 7 - control computer

In mobile agriculture robot control, one of the main aspects is low latency real time video stream. The experiment will be performed by sending video from Raspberry pi to the control computer with different ways and analysed which is faster and with smallest latency. Video stream is performed with 3 different engines. Every stream engine will be tested with nine video resolutions and frame rates (FPS). Each test lasts 10 minutes and is repeated 3 times (total number of experiments 81).

The path from video capture to showing to the control computer can be seen in Figure 2. 


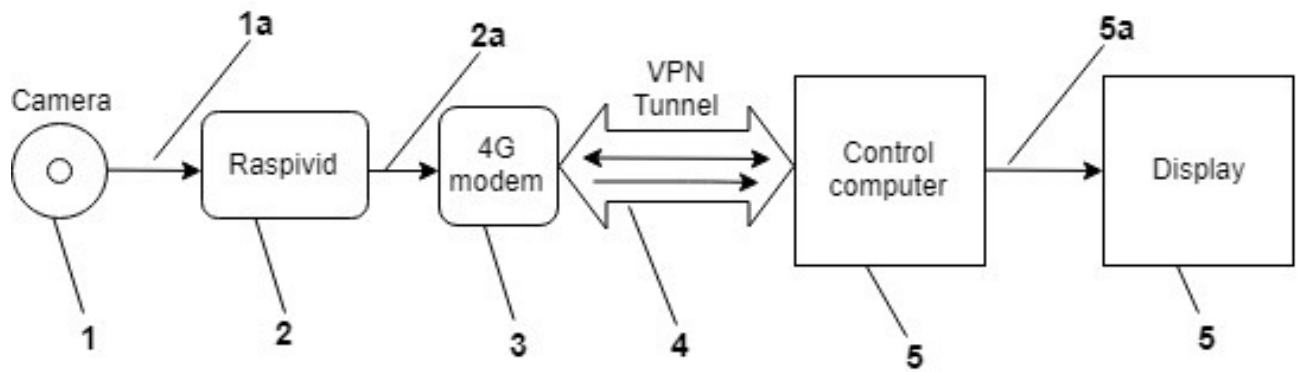

Fig. 2. Video stream scheme: 1 - camera for video; 1a - CSI camera cable 2 - Raspberry Pi video stream engine in Raspberry Pi; 2b- USB connection; 3 - 4G modem Huawei E5377s-32; 4 - OpenVPN tunnel with control link (low bandwidth) and video stream link (high bandwidth); 5 - computer with graphical adapter;

$5 \mathrm{a}$ - computer monitor cable; 6 - display

Table 2

\section{Test methods}

\begin{tabular}{|c|c|c|}
\hline No. & Resolution & Frames \\
\hline 1 & $640 \times 480$ & 10 \\
\hline 2 & $640 \times 480$ & 15 \\
\hline 3 & $640 \times 480$ & 20 \\
\hline 4 & $800 \times 600$ & 10 \\
\hline 5 & $800 \times 600$ & 15 \\
\hline 6 & $800 \times 600$ & 20 \\
\hline 7 & $1024 \times 768$ & 10 \\
\hline 8 & $1024 \times 768$ & 15 \\
\hline 9 & $1024 \times 768$ & 20 \\
\hline
\end{tabular}

\section{Results and discussion}

As you can see in the figures mentioned above, the section results show that Gstreamer and Ffplay are very close to each other and are competitive on various aspects, but VLC is quit out of competition. In real-time video capture less latency is between the capturing device and playback device better, because network latency delays video stream by quite of time. Maximal acceptable latency in this case is $450 \mathrm{~ms}$. If latency is higher than $450 \mathrm{~ms}$., in this case the agricultural robot cannot get or send commands to the server and, for example, in the worst case scenario the agricultural robot can hit an obstacle and that can cause physical or financial damage.

In Figure 3 and 4 Raspberry Pi Model B CPU and RAM are compared. This comparison is needed, because it affects the whole commanding unit. If there is a system overload in the controlling unit the system will function incorrectly and even give false commands, which could lead to a damage caused to the agricultural robot. In Figure 3 Raspberry Pi Model B CPU usage Gstreamer and Ffplay have shown pretty even results and on maximal testing resolution or $1024 \times 768$ with $20 \mathrm{fps}$ CPU load did not rise above $17 \%$ of total CPU usage, but VLC consumed a lot more computing power with $23 \%$ peak.

In Figure 4 RAM consumption is displayed. As shown, Gstreamer and Ffplay show nearly identical results with $30 \%$ of total RAM consumption, but VLC takes up to $45 \%$ of total RAM consumption.

The Public Utilities commision of Latvia (SPRK) survey shows that maximal average download speed in Latvia is $55 \mathrm{Mbps}$ in $4 \mathrm{G}$ mode, but the authors made various speed tests in Jelgava municipality and the results showed that average maximal download speed is between 10 and $15 \mathrm{Mbps}$, which is more than enough to carry out further testing. As shown in Figure 5, average bandwidth used by comparison of software is between $200 \mathrm{Kbps}$ and $400 \mathrm{Kbps}$. 

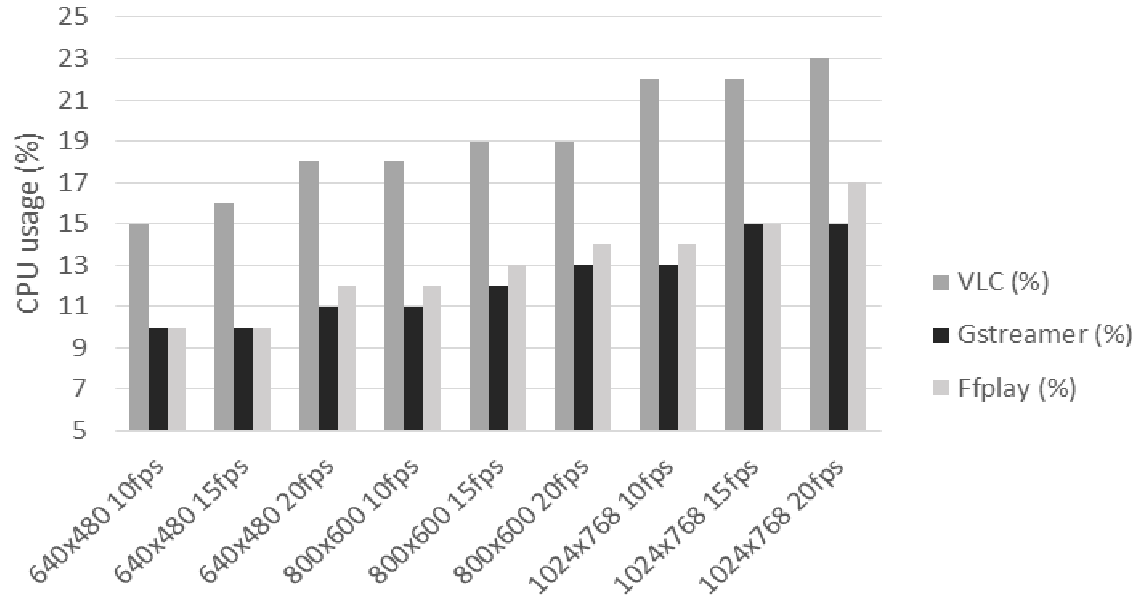

Resolution and frames per second

Fig. 3. Tests for all video stream engines with different resolution and frame rate. CPU usage on Raspberry pi 3

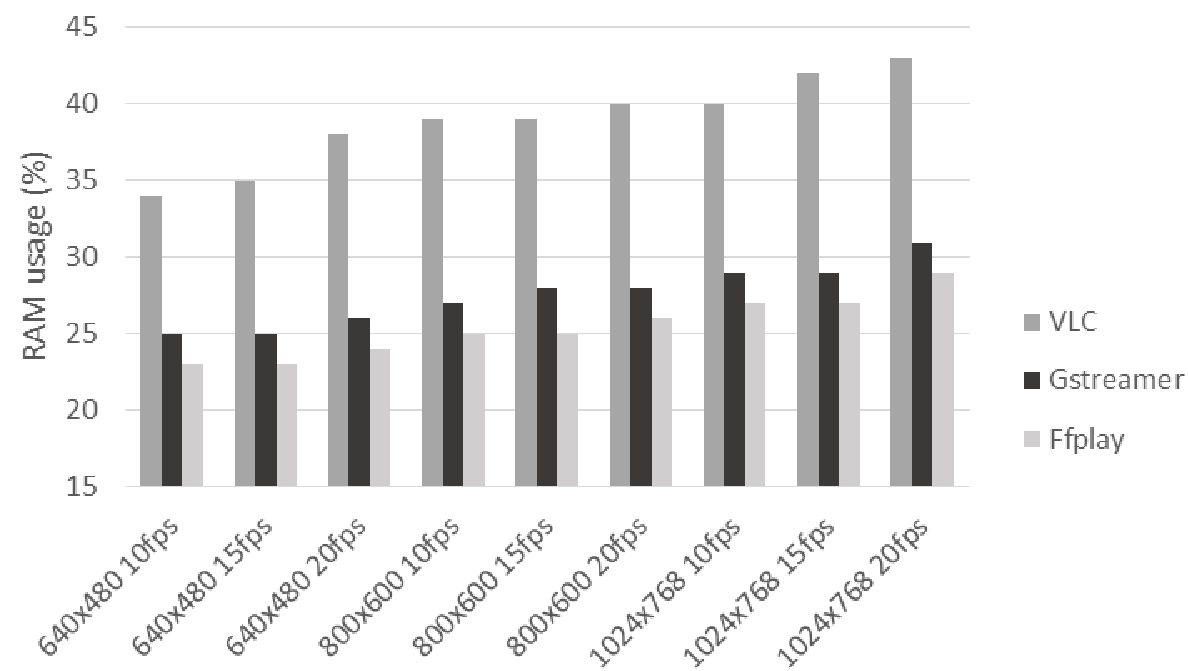

Resolution and frames per second

Fig. 4. Tests for all video stream engines with different resolution and frame rate. RAM usage on Raspberry pi 3

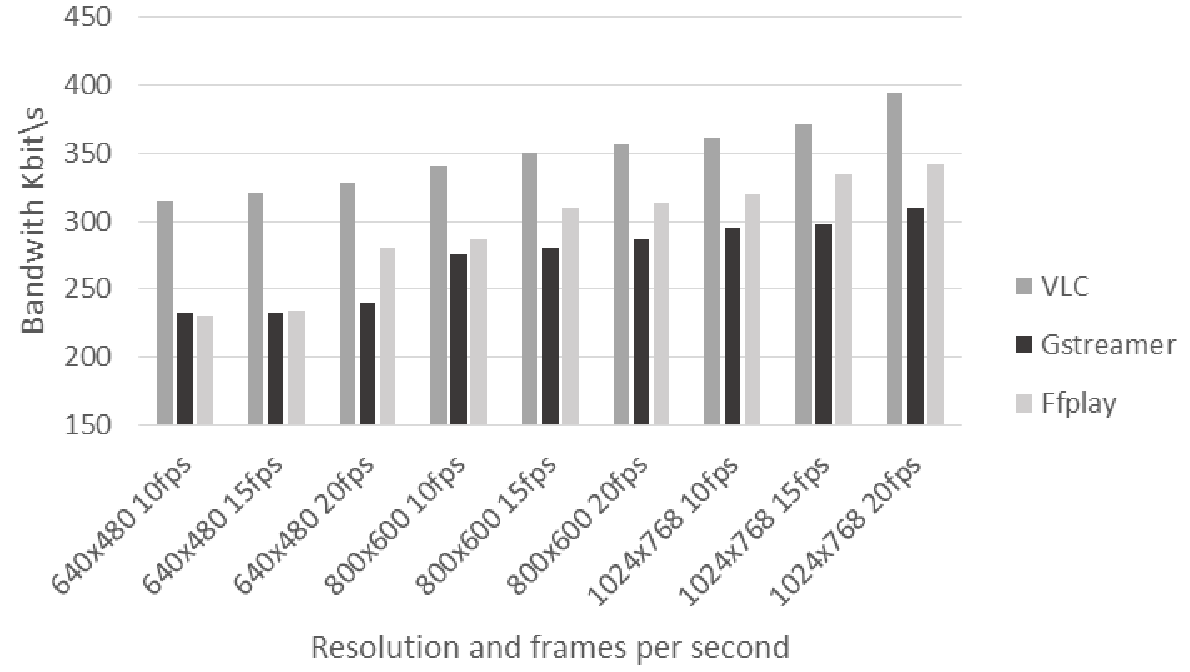

Fig. 5. Tests for all video stream engines with different resolution and frame rate 


\section{Bandwidth usage}

As shown in Figure 6, VLC takes up to seven times more time to carry traffic between the control unit and controlling unit than Gstreamer and Ffplay at maximal testing resulution in 1024x768 with 20 fps. As mentioned before, normal real-time latency between the capture device and playback device is 450 ms., but Figure 6 shows that Gstreamer and Ffplay at 1024x768 resulution with 20 frames per second take up to $1000 \mathrm{~ms}$ to transfer information.

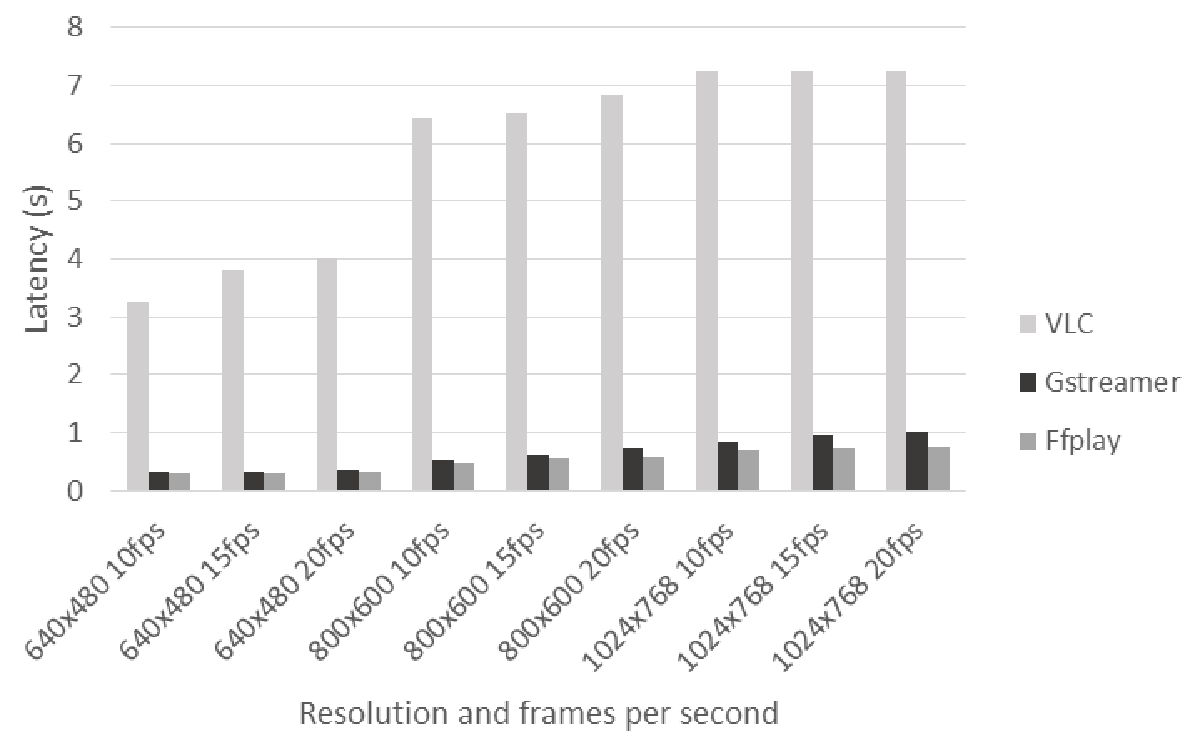

Fig. 6. Tests for all video stream engines with different resolution and frame rate, video latency on control computer

\section{Conclusions}

1. After various conducted tests two out of three softwares were ready for actual real-time video stream and passed all requirements for it.

2. $4 \mathrm{G}$ is capable to transmit real-time data with high bandwidth requirements.

3. $450 \mathrm{~ms}$ is maximal allowed latency to perform real-time video capture and playback.

4. Lower resolution video streams are more accurate and will perform better because of lower latency and need less bandwidth.

This research stated that $4 \mathrm{G}$ is fully capable of carrying real-time data at high bandwidth, but the process can be managed more securely and accurately by using different VPN or other secure connections between devices. The results heavily depend on which network transport protocol (UDP, TCP or RTP) is used and in further research it is needed to get more accurate results.

\section{Acknowledgements}

The authors would like to express their gratitude for the financial support from project "Zinātniskās kapacitātes stiprināšanas LLU” nr. Z18.

\section{References}

[1] Mishra N., Maurya N., Gaur N. Wireless Broadband Network Technology Infrastructure and Related Intellectual Property Application \& Security, 2012

[2] Sabiedrisko pakalpojumu regulěšanas komisijas Elektronisko sakaru kvalitātes pārskats par 2016.gadu (The Public Utilities Commission communication quality report for 2016) [online] [25.04.2018] Available at: https://www.sprk.gov.lv/uploads/doc/Gadaparskats2016FINAL.pdf

[3] Vamsikrishna, P.; Hussain, S.R.; Ramu, N.; Rao, P.M.; Rohan, G.; Teja, B.D.S. Advanced Raspberry Pi Surveillance (ARS) system. In Proceedings of the 2015 Global Conference on Communication Technologies (GCCT), Thuckalay, India, 23-24 April 2015; pp. 860-862. 
[4] Boyaci O., Forte A., Baset S.A., Schulzrinne H. vDelay: A tool to measure capture-to-display latency and frame rate. In Proceedings of the 11th IEEE International Symposium on Multimedia, San Diego, CA, USA, 14-16 December 2009; pp. 194-200.

[5] Jennehag U., Forsstrom S., Fiordigig F.V. Low Delay Video Streaming on the Internet of Things Using Raspberry Pi, 2016

[6] Yakova Z. A New Virtual Private Networks Access Model Doctoral Conference in Mathematics, Informatics and Education, At Sofia, Bulgaria, Volume: 1, September 2013; pp 125-130

[7] Che Fai Y., Amin S.H.M., Fisal N., Abu Bakar J. "Bluetooth Enabled Mobile Robot." IEEE lCIT'02, Bangkok, THAILAND 2002.

[8] Cleveland F., Uses of the New Types of Wireless Technologies for Distribution and Substation Automation, , Xanthus Consulting International, 2007.

[9] Raspberry Pi 3 Model B [Online] [21.03.2018] Available at: https://www.raspberrypi.org/products/raspberry-pi-3-model-b/

[10] OpenVPN security [Online] [21.03.2018] Available at: https://openvpn.net/index.php/accessserver/security-advisories.html 